\title{
Ultra-high energy neutrino search with the Askaryan Radio Array
}

\section{Ming-Yuan Lu ${ }^{* a}$, Carl Pfendner $^{b}$, and Andrew Shultz ${ }^{c}$}

${ }^{a}$ Wisconsin IceCube Particle Astrophysics Center (WIPAC), University of Wisconsin-Madison, Wisconsin, U.S.A.

${ }^{b}$ Department of Physics and Center for Cosmology and Astro-Particle Physics, The Ohio State University, Ohio, U.S.A.

${ }^{c}$ Department of Physics and Astronomy, University of Nebraska-Lincoln, Nebraska, U.S.A.

E-mail: mlu27@icecube.wisc.edu

\section{for the ARA Collaboration}

Full author list: http://ara.wipac.wisc.edu/collaboration/authors/ara_icrc17

The Askaryan Radio Array (ARA) is an ultra-high energy neutrino telescope aiming to discover and determine the cosmic neutrino flux above $\sim 100 \mathrm{PeV}$. Under phased construction near the South Pole, ARA achieves the sensitivity through the detection of Askaryan emissions from neutrino-induced showers in the Antarctic ice using radio frequency antennas $(150-850 \mathrm{MHz})$. Two ARA stations at a design depth of $200 \mathrm{~m}$ have been deployed since 2013 . We present in this contribution various neutrino search methods developed, and the search results using data from 2013 and after.

35th International Cosmic Ray Conference - ICRC2017

10-20 July, 2017

Bexco, Busan, Korea

${ }^{*}$ Speaker. 


\section{Introduction}

First predicted by Greisen, Zatsepin, and Kuzmin in 1966 [1,2], ultra-high energy cosmic rays (UHECR) more energetic than $10^{19.5} \mathrm{eV}$ are expected to engage in photopion production with the cosmic microwave background (CMB) photons to yield an almost guaranteed flux of UHE neutrinos. This "cosmogenic" neutrino flux has yet to be discovered. Measurement of this neutrino flux will provide exclusive insights into the UHE cosmic ray composition, source evolution, and source location.

However, the weakly interacting nature of neutrinos and the low flux of UHECR pose fundamental difficulties to detecting such neutrinos, as the flux level is $\sim<1$ event $/ \mathrm{yr} / \mathrm{km}^{3}$. This requires a ground-based detector covering $O(100 \mathrm{~km})$. We plan to achieve this detector area by observing the broadband radio-frequency (RF) signal emitted from neutrino-induced particle showers in dense media through the Askaryan effect [3]. We choose South Pole as the experiment site due to its large masses of RF-transparent ice, where the attenuation length is typically of order $\sim 1 \mathrm{~km} \mathrm{[4].}$ Therefore, essentially by combining RF detection with large volumes of natural target mass, we are able to build a cost-effective UHE neutrino telescope - the Askaryan Radio Array (ARA) (Fig.1). The completed ARA will cover $\sim 100 \mathrm{~km}^{2}$, and is able to measure the wavefront, the polarization, and the frequency content of the described Askaryan signal.

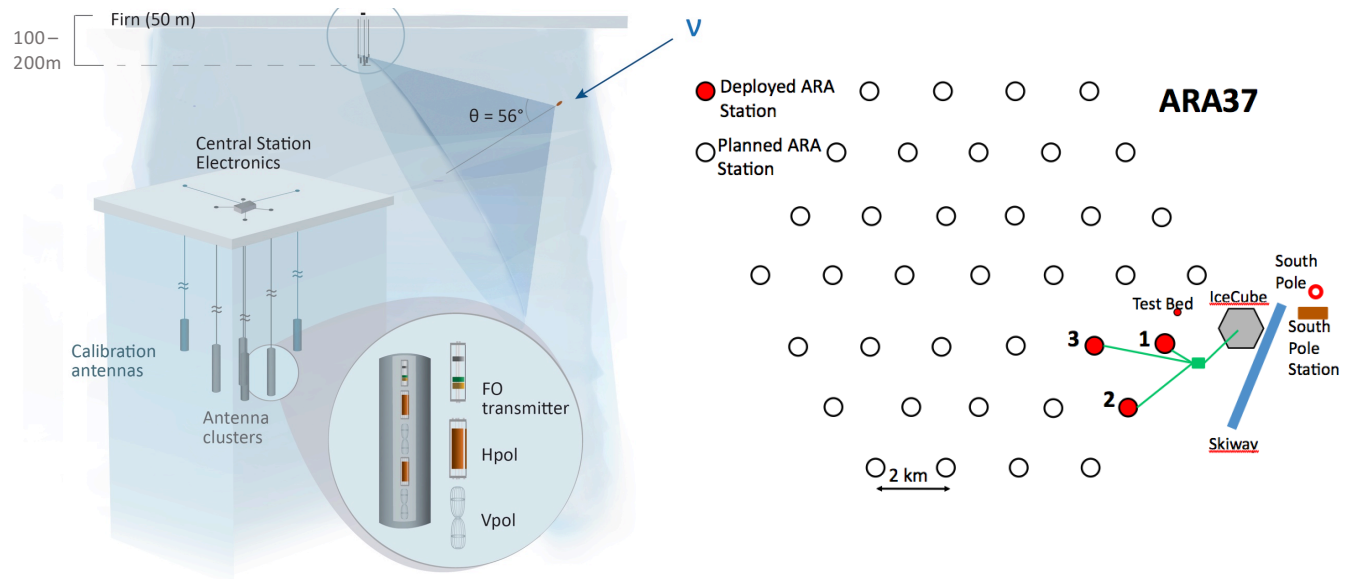

Figure 1: Left: neutrino detection with ARA. A neutrino-induced shower emits Askaryan signal near the Cherenkov angle. In an ARA station, four central strings connect the measurement antennas. Two outer strings connect the calibration pulsers. Right: baseline ARA with 37 stations.

\section{The ARA Detector}

The baseline ARA consists of 37 antenna clusters (stations) on a hexagonal grid (Fig.1). Each station is equipped with 16 downhole measurement RF antennas installed on four $200 \mathrm{~m}$ strings on a circle of diameter $20 \mathrm{~m}$, and 4 calibration pulsers on 2 strings $\sim 40 \mathrm{~m}$ away from a station center. Measurement antennas are either vertically polarized (Vpol) or horizontally polarized (Hpol). These antennas are designed to be broadband and azimuthally symmetric. The registered signals on downhole antennas are bandpass filtered between $150 \mathrm{MHz}$ to $850 \mathrm{MHz}$, and notched filtered at 
$450 \mathrm{MHz}$ to eliminate out-of-band and narrow-band South Pole communications frequencies. Signals are then amplified, converted to optical signals, and transmitted to the surface, where the data acquisition (DAQ) box sits. At the DAQ box, the signals are converted back to electric analog, and are split into two paths - trigger and digitization. Implemented by an on-board field programmable gate array (FPGA), the trigger logic searches for three coincident power excursions over a dynamic threshold among eight electric channels of the same polarization in a preset time frame. The threshold is servo-ed to keep a trigger rate of approximately $5 \mathrm{~Hz}$ from thermal backgrounds, which dominates ARA trigger. Once triggered, digitization with the IRS2 ASIC on all channels is carried out at 3.2GS/s. Such a collection of digitized waveforms constitutes an event. For details, see [5]. Currently there are three deployed ARA stations, and we expect to add three more in the upcoming Pole season.

\section{Simulation}

We use the simulation program AraSim, to generate thermal backgrounds and neutrino events to characterize our analysis techniques. AraSim models the signal generation, the signal propagation, and the instrumental response of an ARA station to produce Monte Carlo events in the same format as real data. At the signal generation stage, independent interaction vertices with random momentum directions are randomly generated around a single station in a cylindrical simulation volume. The flavor, interaction type, and energy can be specified by the AraSim user. The Askaryan emission is then modeled by a parametrized shower model and its RF emission [6]. In the propagation stage, the signal is attenuated according to its path through the ice, where a depth-dependent index of refraction model [7] is used. The ray-bending effect due to the inhomogeneous ice is characterized by a semi-analytic algorithm implemented with a custom code RaySolver [8]. Lastly, in the instrumental response stage, we convolve the signal with the antenna response, and apply the filter and amplifier responses. Here, thermal background waveforms generated by Rayleigh fits to past thermal data are added to the signal [8]. The trigger logic is identical to that of a real station. Once the trigger condition clears, waveforms are read out.

\section{Data Analysis}

An ARA analysis usually involves a set of event selection criteria and a neutrino reconstruction technique. The event selection rules need to exclude data from faulty periods of the electronics, or when non-physics background dominates our trigger. Most importantly, thermal backgrounds should be rejected by a factor of $\sim 10^{-10}$ [5], at our current trigger rate. Events that survive the selection criteria will be reconstructed. As a first step, we attempt to reconstruct the vertex direction with respect to the station. Vertex distance and neutrino momentum direction, though out of scope for this contribution, can then in principle be reconstructed by measuring the curvature of the signal wavefront and the polarization. Further event selection cuts can be placed using information from the reconstruction. For example, we can devise reconstruction quality cuts and geometric cuts that exclude events from above the ice surface or known anthropogenic sources. This contribution will discuss filter and reconstruction algorithms developed in parallel, with a focus on the radiospline interferometric reconstruction method. This method utilizes radiospline to determine delays used 
in constructing event-by-event interferometric skymaps. This allows us to take into account the aforementioned ray-bending effect when reconstructing.

\subsection{Radiospline Interferometric Reconstruction}

In an interferometric reconstruction, we assume a set of putative vertex locations. For each location, the signal arrival times (delays) to antennas are calculated. Taking any pair of antennas (a baseline), we compute the cross correlation (CC) value according to the difference in delays. We define the coherence value as the sum of the $\mathrm{CC}$ values from all same-polarization baselines after normalizing for channel-to-channel power level differences and number of baselines used. Here, we define the putative vertex locations as the pixel centers of a collection of Healpix [9] skymaps centered around our station at different radial distances. All skymaps shown in this contribution have 196,608 pixels (mean pixel spacing $0.4581^{\circ}$ ). For neutrino reconstruction, 50 skymaps at radial distances from $100 \mathrm{~m}$ to $5000 \mathrm{~m}$ in $100 \mathrm{~m}$ steps are generated. Delays are computed with Radiospline [10]. The use of Radiospline readily accounts for the ray-bending effect in this reconstruction technique. Note that in this contribution we use only the Vpol baselines for reconstruction. A Vpol n-channel filter requiring at least three channels with peak voltage above a predetermined threshold is applied before event reconstruction. A threshold scan on the yearly $10 \%$ unblinded RF sample gives the threshold that yields $1 \%$ thermal noise rejection.

\subsubsection{Pulser Reconstruction}

Lacking physics background, ARA depends on man-made sources for calibration. Reconstructing the calibration pulsers installed on ARA stations by fixing the skymap radial distance to $42 \mathrm{~m}$, the known calibration pulser distance, in general we can reconstruct the pulser direction to $\sim<1^{\circ}$ accuracy. Other calibration sources include radio transmitters deployed along IceCube strings 1 and 22, called the deep pulsers. Each string sits approximately four kilometers away from ARA, and hosts two transmitters installed at depths of $1450 \mathrm{~m}$ and $2450 \mathrm{~m}$ below the ice surface. An ARA3 reconstruction skymap of the 1450m deep pulser on string 22 is shown in Fig.2. The systematic offset of the hotspot from the expected direction and the ice properties inferred are discussed in [11]. Note that the deep pulsers are situated in the geometric phase space to which ARA is most sensitive. A reconstruction to deep pulser signals thus represents the best proxy for ARA's ability to reconstruction neutrinos.

\subsubsection{Simulation Reconstruction}

We produce AraSim data for neutrino energies from $10^{16}$ to $10^{21} \mathrm{eV}$ in full energy decades to study the event selection and the reconstruction. Fig.3 demonstrates our ability to reconstruct vertex direction to a zenith resolution of $1.3^{\circ}$ and a azimuth resolution of $0.31^{\circ}$ using radiospline interferometry. With the multi-layer skymap approach described earlier, we are not able to resolve the vertex distance. This, combined with ray-bending, limits us from attaining sub-degree zenith resolution.

\subsubsection{Reconstruction Quality Cut}

The interferometric approach has the advantage of averaging down uncorrelated thermal noise. Therefore, the coherence value can be viewed as a reconstruction quality parameter and be used to 


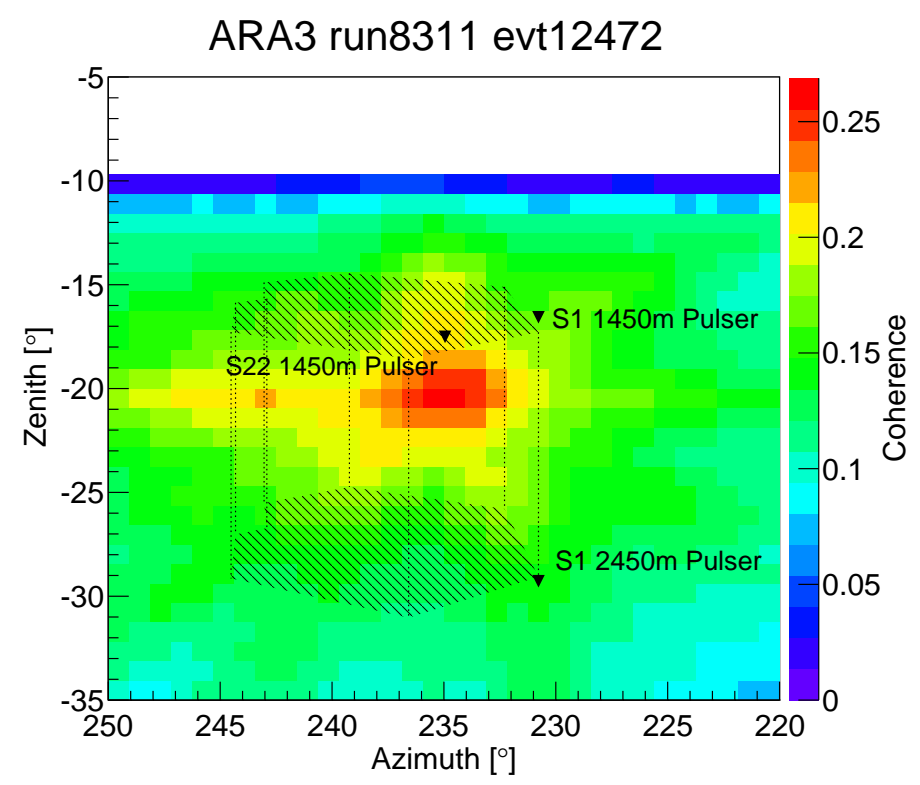

Figure 2: Partial reconstruction skymap of the IceCube deep pulser (2015 string 22 depth: 1450m) with ARA3.The overlaying hexagonal prism is the IceCube detector as seen by ARA3. The hotspot in coherence defines the reconstruction direction. The skymap radial distance is set equal to the true distance $(4040 \mathrm{~m})$ between ARA3 and the pulser in use. Note that the coherence values here are normalized differently than shown in other figures as a later effort to account for channel multiplicity.
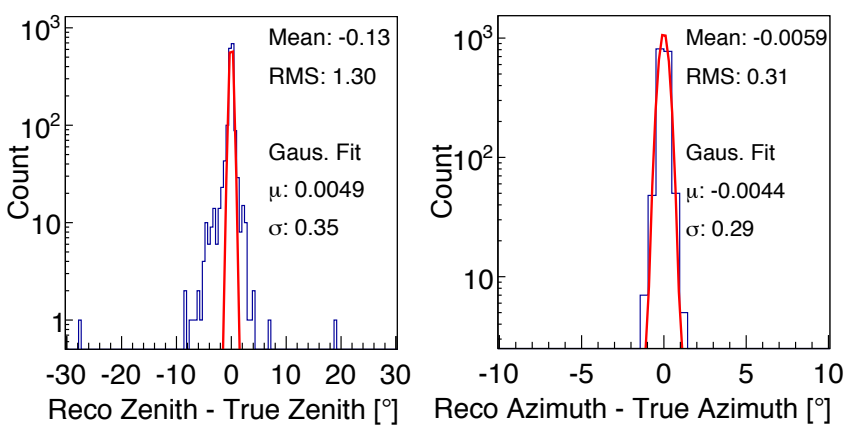

Figure 3: Simulated neutrino interaction at $10^{18} \mathrm{eV}$ vertex direction reconstruction. Plots show the difference of reconstructed zenith/azimuth from true values after maximal coherence value $>5.0$ cut. The fitting curves represent Gaussian fits to the histograms.

reject thermal events. The cut efficiency for Monte Carlo mono-energetic neutrinos against thermal noise is showcased in Fig.4. We use an exponential fit to the noise tail to extrapolate to a cut value, 0.1082 , corresponding to the desired noise pass rate of $10^{-8}$. At $10^{18} \mathrm{eV}, 53.1 \%$ of the signal events are retained. The cut is currently being further studied.

\subsection{Other Filters in Development}

Several other filter techniques have been studied. These include the time sequence filter [5], 


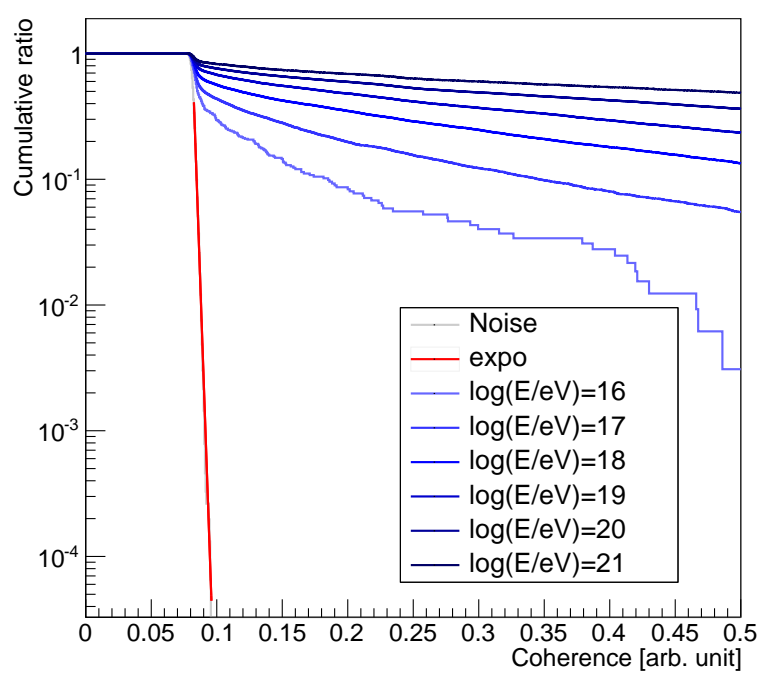

Figure 4: Cumulative ratio of simulated noise and signal events versus coherence cut. The gray line (Noise) is the simulated noise pass rate. The red line represents the exponential fit to the noise tail. Fitted equation: $y=e^{55.4885-682.864 x}$. The cut value at $10^{-8}$ is 0.1082 .

a modified $\mathrm{n}$-channel filter that uses the Hilbert envelope instead of raw waveforms to determine the voltage threshold, the wavefront root-mean-square (RMS) method, and the surface filter. We will introduce the latter two. The wavefront RMS method takes advantage of the regular geometry of the ARA station and places constraints on the arrival times of the signal. Assuming that the incoming signal can be approximated as a plane wave, timing calculations can be used to reject signals that deviate from this assumption. First, one finds the peak of each waveform after a 5-ns power integration, so long as that peak passes an SNR threshold. After normalizing for the distance between the antennas, one compares the hit times in similar pairs of antennas (e.g. vertically aligned pairs) by finding the RMS of the delays in each pair. This "wavefront RMS" is an estimator for how closely the arrival time differences agree with each other and can be used as a tunable cut parameter. As seen in Fig.5, the method provides a potential improvement over the time sequence quality parameter used in [5]. The cut values used for each method give identical rejection factors (99.92\% rejection) for RF noise data and overall simulated neutrino acceptance improves from $81.6 \%$ to $83.1 \%$ by using the wavefront RMS method.

The surface filter is based on the minimum arrival angle criterion of signals to suppress backgrounds from the ice surface. The wavefront from sources above the ice refracts at the surface and is further curved due to the changing index of refraction, resulting in the arrival angles of $\leq 35^{\circ}$ to vertical, while in-ice neutrino interactions typically result in larger arrival angles. The arrival angle is reconstructed with the analytic spheres method (ASM), which consists of solving three equations with three unknowns $(\mathrm{x}, \mathrm{y}, \mathrm{z})$ of the source position that compare the observed and expected time differences between the channels given the geometry and the ice model for every possible combination of four hits in an event, and then averaging across all results. The resolution in the zenith angle is $2.5^{\circ}$. This filter has been evaluated using an AraSim sample of neutrinos generated 
at $10^{18} \mathrm{eV}$ and a sample of point-like radio sources generated with a uniform spatial distribution above-ice in the few-kilometer vicinity of an ARA station. The reconstructed arrival angles show a very good separation between in-ice neutrino vertices and above-ice radio sources (Fig.5).
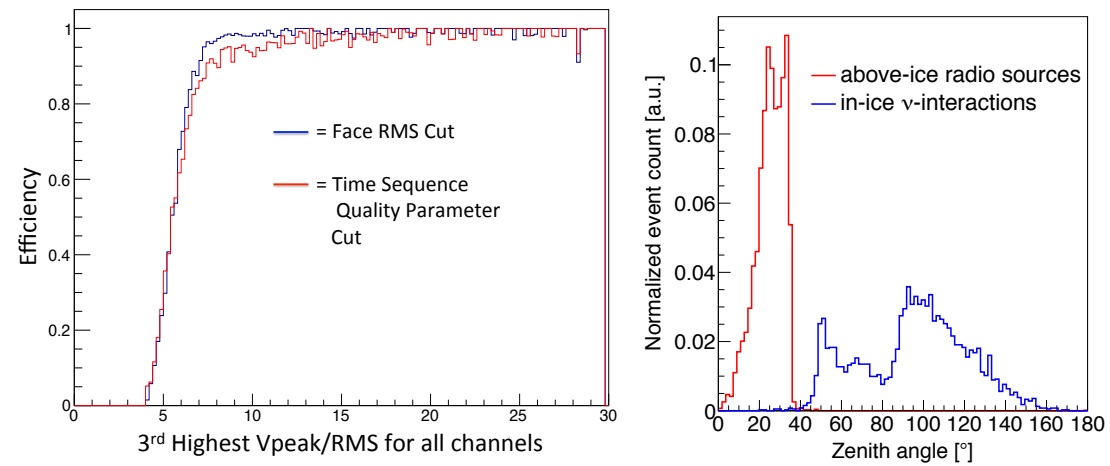

Figure 5: Left: the efficiency as a function of signal strength for the wavefront RMS method and the time sequence quality parameter for a simulated set of neutrinos generated at $10^{18} \mathrm{eV}$. Right: reconstructed wavefront arrival angles originating from neutrino interactions (blue) and above-ice radio sources (red). Event count is normalized by the total event number.

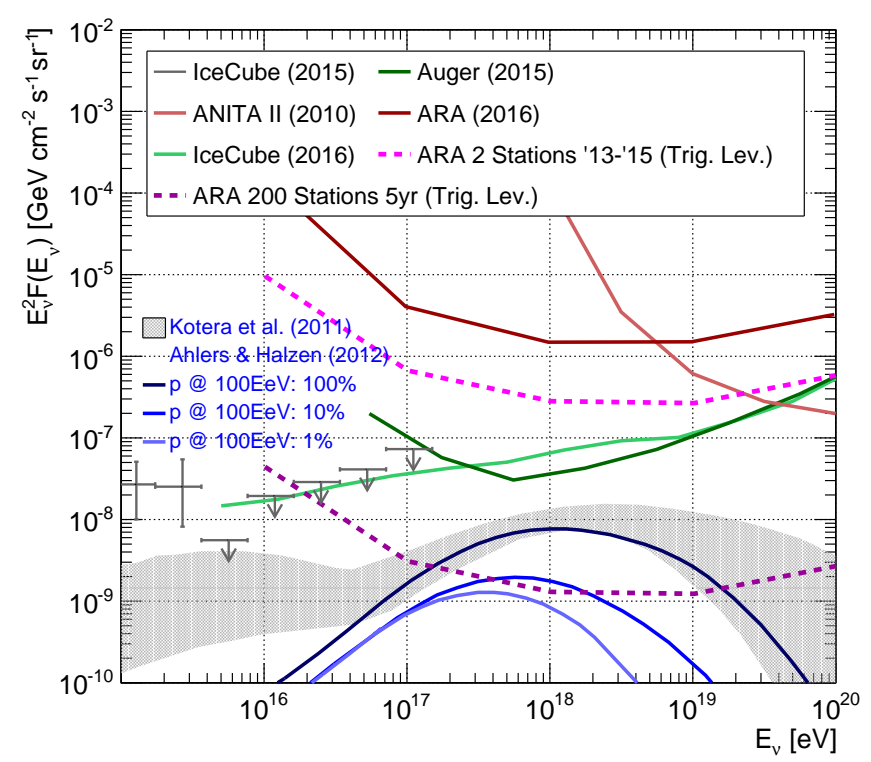

Figure 6: The projected trigger level sensitivity of this work (ARA 2 Stations '13-'15), as compared to previously published ARA upper limit (ARA (2016) [5]). The current best limits are set by IceCube, Auger, and ANITA [14-17]. A projected sensitivity of 200 ARA statiosn in five years demonstrates the potential of a radio array to probe cosmogenic flux models $[18,19]$ down to $E^{2} F\left(E_{v}\right)=10^{-9} \mathrm{GeV} \mathrm{cm}^{-2} \mathrm{~s}^{-1} \mathrm{sr}^{-1}$.

\section{Summary and Outlook}

We presented filter techniques that efficiently reduce thermal background and the radiospline 
interferometric technique that reconstructs various calibration sources and simulated neutrino signals to $\sim 1^{\circ}$ angular resolution. Additional filter algorithms are also discussed. We plan to fully characterize these techniques, and potentially combine them in a complementary manner to reach maximal neutrino sensitivity. Fig.6 shows our combined trigger level sensitivity with 2013-2015 data from ARA2 and ARA3, with live times 28.2 months and 27.2 months each. Despite not being the most sensitive in the GZK regime yet, an ARA-like detector allows cost-effective scaling of the detector area to reach physically significant sensitivities, as demonstrated by the projected sensitivity of 200 ARA stations in five years. On top of this, optimization studies have found an increased sensitivity when doubling the station geometry, e.g. by $25 \%-30 \%$ at $10^{18} \mathrm{eV}$ [12]. Moving forward, our calibration studies will not only enhance understanding of the ice properties but also provide pointers to future radio detector design, and unblinding the 2013-2015 two stations data will update our upper limit in the case of null observation. By the end of early 2018, there will be six ARA stations. The addition of a phased array string to one ARA station this Pole season holds promise to increase the sensitivity of that station by a significant factor especially at low energies. With verified design, deployment, and data analysis, the future ARA will be a promising candidate in detecting UHE neutrinos.

\section{References}

[1] K. Greisen, Phys. Rev. Lett. 16748 (1966)

[2] G. T. Zatsepin and V. A. Kuzmin, JETP Lett. 478 (1966).

[3] G. A. Askaryan, JETP 14, 441 (1962).

[4] P. Allison et al. (ARA), Astropart. Phys. 35457 (2012).

[5] P. Allison et al. (ARA), Phys. Rev. D 93082003 (2016).

[6] J. Alvarez-Muniz, A. Romero-Wolf, E. Zas, Phys.Rev. D 81123009 (2010).

[7] I. Kravchenko, D. Besson, J. Meyers, Journal of Glaciology 50 522-532 (2004).

[8] P. Allison et al. (ARA), Astropart. Phys. 7062 (2015).

[9] Gorski, K. M. et al., ApJ 622759 (2005).

[10] M. Beheler-Amass et al., in proceedings of the 35th ICRC (2017), POS (ICRC2017) 1054.

[11] D. Seckel et al. (ARA), in proceedings of the 35th ICRC (2017).

[12] R. Khandelwal, M.-Y. Lu, A. Karle, in proceedings of the 35th ICRC (2017), PoS (ICRC2 017 ) 938.

[13] A. G. Vieregg, K. Bechtol, A. Romero-Wolf, JCAP 2005 (2016).

[14] M. G. Aartsen et al. (IceCube Collaboration), in proceedings of the 34th ICRC (2015), (2015).

[15] A. Aab et al. (Pierre Auger Collaboration), Phys. Rev. D 91092008 (2015).

[16] P. W. Gorham et al., Phys. Rev. D 85049901 (2010).

[17] M. G. Aartsen et al. (IceCube Collaboration), Phys. Rev. Lett. 117241101 (2016).

[18] K. Kotera, and A. V. Olinto, Annu. Rev. Astron. Astrophys. 49119 (2011).

[19] Ahlers and Halzen, Phys. Rev. D 86083010 (2012). 